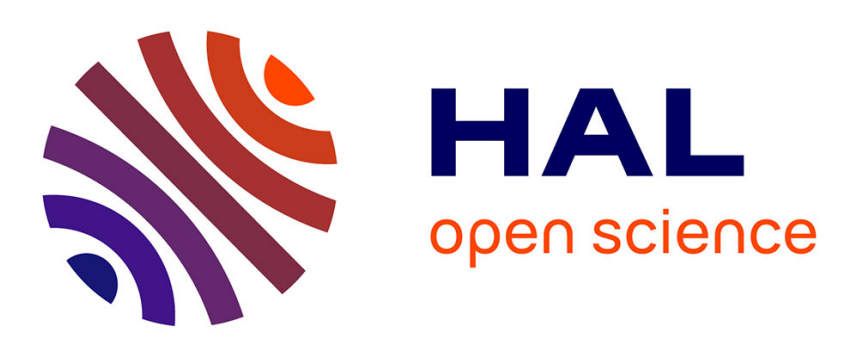

\title{
Regularized non negative matrix factorization for autofluorescence removal in fluorescence optical imaging
}

Anne-Sophie Montcuquet, Lionel Herve, Fabrice Navarro, Jean-Marc Dinten, Jerome I. Mars

\section{- To cite this version:}

Anne-Sophie Montcuquet, Lionel Herve, Fabrice Navarro, Jean-Marc Dinten, Jerome I. Mars. Regularized non negative matrix factorization for autofluorescence removal in fluorescence optical imaging. SSP 2011 - 2011 IEEE Workshop on Statistical Signal Processing, Jun 2011, Nice, France. pp.P001. hal-00610052

\section{HAL Id: hal-00610052 \\ https://hal.science/hal-00610052}

Submitted on 21 Jul 2011

HAL is a multi-disciplinary open access archive for the deposit and dissemination of scientific research documents, whether they are published or not. The documents may come from teaching and research institutions in France or abroad, or from public or private research centers.
L'archive ouverte pluridisciplinaire HAL, est destinée au dépôt et à la diffusion de documents scientifiques de niveau recherche, publiés ou non, émanant des établissements d'enseignement et de recherche français ou étrangers, des laboratoires publics ou privés. 


\title{
REGULARIZED NONNEGATIVE MATRIX FACTORIZATION FOR AUTOFLUORESCENCE REMOVAL IN FLUORESCENCE OPTICAL IMAGING
}

\author{
Anne-Sophie Montcuquet ${ }^{1,2}$, Lionel Herve ${ }^{1}$, Fabrice Navarro ${ }^{1}$, Jean-Marc Dinten ${ }^{1}$ and Jérôme I. Mars ${ }^{2}$ \\ ${ }^{1}$ CEA, LETI, MINATEC, 17 rue des martyrs, 38054 Grenoble cedex 9, France \\ ${ }^{2}$ GIPSA-Lab, Rue de la houille blanche BP46, 38402 Saint Martin d'Hères, France
}

\begin{abstract}
Fluorescence imaging in diffusive media locates cancers thanks to injected fluorescent markers specific to the tumors. The region of interest is illuminated with red light and the emitted back fluorescence is analyzed to locate the fluorescence sources. To detect accurately the markers signal and be able to explore thick media (breast, prostate), autofluorescence emitted by biological tissues has to be removed. We propose a spectroscopic approach, based on Non-negative Matrix Factorization (NMF) method, and present interest of regularized NMF algorithms on unmixing results. In vivo autofluorescence removal and tumor detection enhancement results on mice will be presented.
\end{abstract}

Index Terms - Fluorescence spectroscopy, autofluorescence, positive source separation

\section{INTRODUCTION}

In fluorescence imaging, specific fluorescent markers are injected to a patient to specifically bind to biological targeted such as tumors [1]. The region of interest is then illuminated with near infrared (NIR) light. An optimal wavelength range may be defined - between 600 and $900 \mathrm{~nm}$ - where the tissue absorption is lower: it allows light to excite markers around tumors deep (a few centimeters) in tissues. Finally, the emitted back fluorescence signal is measured and the tumors are localized by fluorescence optical tomography (FDOT). As NIR light excites injected fluorescent markers, it also excites all fluorophores naturally present in tissues: this unwanted measured signal is called autofluorescence. To investigate thick media for medical diagnostic application (breast or prostate cancer diagnosis for example), as the fluorescence signal gets exponentially weak with the light travel distance, the autofluorescence of tissues becomes a limiting factor. The analysis of a fluorescence signal impaired by autofluorescence may lead to a wrong localization of the markers: the signal needs to be preprocessed in order to remove autofluorescence. For in vivo fluorescence spectroscopy, the unmixing problem is referred to as a blind source separation problem since the fluorescent sources spectra may vary according to the fluorescent dye biological environment. Fluorescence spectra to separate are also supposed statistically dependent, which filters out many methods (such as ICA). Non-negative Matrix factorization (NMF) differs from classical source separation methods (SVD, PCA) in that it forces the matrices factors to be non-negative, and thus suits to spectra separation.
As in many cases, the obtained solution is non-unique, and prior information is necessary to restrain the NMF solution set. We developped new regularized NMF algorithms (initialisation of problem, and spatial sparsity constraints) which bring prior information to the unmixing problem and improve NMF results. Finally, regularized methods are tested and validated on in vivo mice data.

\section{NON-NEGATIVE MATRIX FACTORIZATION}

The classical NMF definition says[2]:

Given a nonnegative matrix $V \in \mathbb{R}^{N_{s} \times N_{\lambda}}$, find nonnegative matrices $A \in \mathbb{R}^{N_{s} \times P}$ and $S \in \mathbb{R}^{P \times N_{\lambda}}$ such that :

$$
V \simeq A S
$$

where nonnegative matrices are matrices whose all factors are nonnegative and $P$ stands for the number of sources to unmix.

Applied to spectroscopy, matrix $A$ is considered as the weights matrix, and $S$ as the spectra matrix, both containing as much elements $P$ as fluorescent sources to separate. Note that our model does not include noise since in our system measurement noise is very low. To find the particular matrices $A$ and $S$ that satisfy equation 1, we define the following cost function to minimize:

$$
F=\|V-A S\|_{2}^{2}
$$

Then to minimize $F$, after matrices $A$ and $S$ initialization with positive values, multiplicative update rules developped by Lee and Seung [2] are iteratively applied. It brings us to the following NMF implementation:

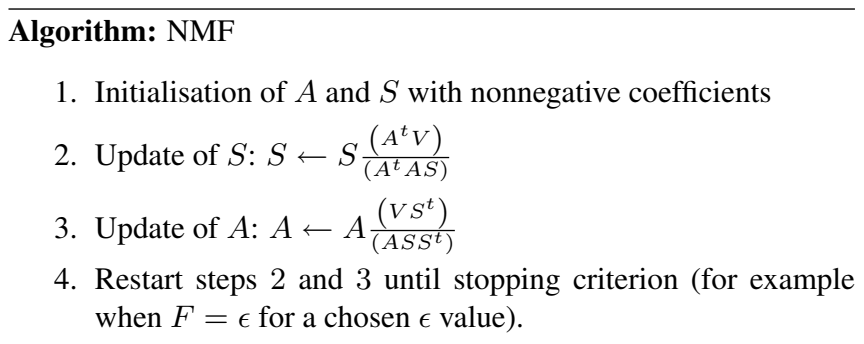

\section{REGULARIZATION}

\subsection{Non-uniqueness of solution}

The chosen cost function $F$ is not jointly convex in matrices $A$ and $S$ : there are numerous local minima to the function and nonuniqueness of the NMF factorization. Let us assume a factorization 
of $V$ by the product of some matrices $A$ and $S$ exists. If we now consider any invertible matrix $T$ of size $P \times P$, then a new couple $(\tilde{A}, \tilde{S})$ of solutions is easily found:

$$
V=\underbrace{\left(A T^{-1}\right)}_{\tilde{A}} \underbrace{(T S)}_{\tilde{S}}
$$

Without any constraint and prior information on sources, there is an infinity of factorizations of matrix $V$. Non-negativity constraint inherent to the NMF problem already restrains the solution set [3]. But other regularization constraints may be added to original cost function $F$, and prior information may be considered to improve the unmixing [4]. We propose to study the influence of initialization of $A$ and $S$ on NMF decomposition, and to add spatial sparsity constraints to the unmixing problem. To underline regularization effect, we compare NMF results with or without regularization on simulated breast fluorescence data [5].

\subsection{Simulated example}

Many clinical experiments on breast allow us to design a computer breast model with realistic tissues optical properties. A fake marked tumor is introduced to the model, and consistent modeling fluorescence acquisitions of the simulated breast are obtained, with modified depth of the marked tumor.

Definition of simulated matrices $A$ and $S$ : The breast fluorescence acquisition model is composed of an homogeneous autofluorescence distribution and of a specific fluorescence contribution (to mimic the tumor pointed out by an injected fluorescent marker). Signal ratio between healthy tissue and tagged tumor depends on biomarkers. From bibliography, and from experience, ratios from 3 to 15 [6] (for more specific-to-tumor markers) are usual. We chose for our simulation study a ratio between tumor and healthy tissue approximately equal to 10 when tumor is $1 \mathrm{~mm}$ deep in tissues.

The specific fluorescence part is the product of a weight vector $A_{1}$ by a fluorescence spectrum $S_{1}$ (see figure 1 - a). In the same way, the autofluorescence part is the product of the weight vector $A_{2}$ by a fluorescence spectrum $S_{2}$ (see figure 1 - b). Simulated spectra are gaussian models chosen close to usual fluorescence spectra of autofluorescence and fluorescent markers (Indocyanine Green for example). Finally the total simulated acquisition is obtained by adding the specific fluorescence and the autofluorescence parts (figure 1 - c).

Contrast definition: We introduce the contrast $C_{T, N}$, measured between tumorous area $T$ and normal tissues area $N$ : it characterizes the improvement of tumor detection after autofluorescence removal, on simulation and experimental results. Average intensity of fluorescence signal is measured on both concerned areas: $\bar{T}$ and $\bar{N}$ are respectively the average intensities in photons per pixel of areas $T$ and $N$ defined figure 1, and: $C_{T, N}=(\bar{T}-\bar{N}) /(\bar{T}+\bar{N})$. The closer to one the contrast value gets, the better the detection will be. On next regularization tests, simulated marked tumor is moved from surface to $5 \mathrm{~cm}$ deep in tissues, and contrast between tumor and healthy tissue is calculated for each position.

\subsection{Influence of initialization choice on NMF decomposition}

NMF decomposition directly depends on the initial guess on matrices $A$ and $S$. We study influence of initialization on our simulated

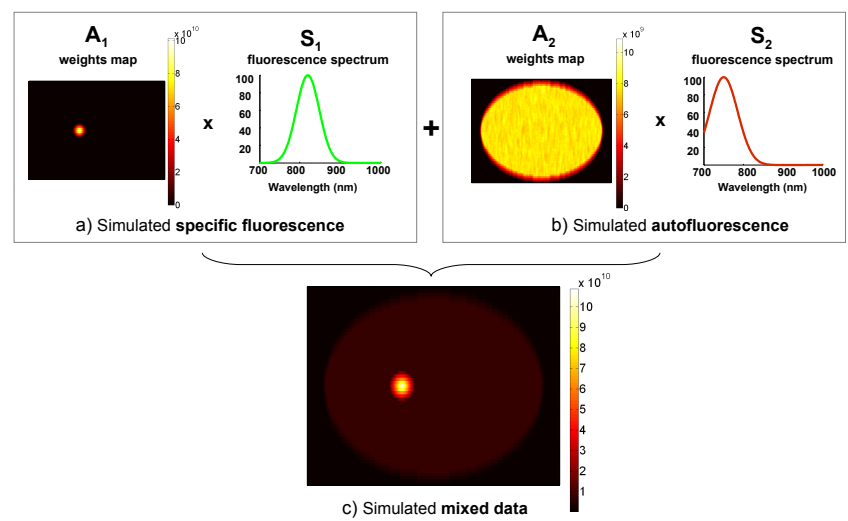

Fig. 1. The sum of a specific fluorescence signal a) and an autofluorescence signal $\mathbf{b}$ ) leads to simulated mixed data $\mathbf{c}$ ).

example: Gaussian spectra similar to simulation spectra of matrix $S$ are chosen to initialize the NMF algorithm, but a range of translated initialization spectra is tested. Initialization spectra for matrix $S_{0}$ are translated on a range of $100 \mathrm{~nm}$, on both sides of simulation spectra, as depicted figure 3.3 .

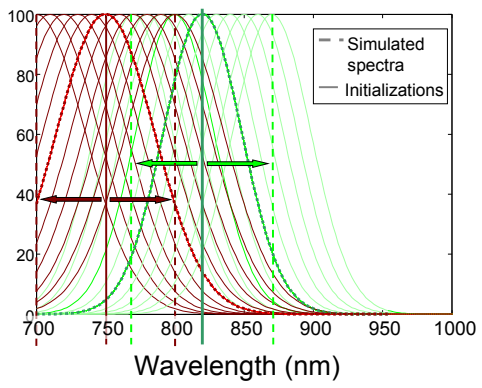

Fig. 2. Influence of $S_{0}$ initialization translation on NMF decomposition.

First we observe healthy tissue/tumor contrast on raw data, without any unmixing processing: contrast and detection are naturally decreasing with depth (see figure 3 - a). Then NMF processing is applied on data but with random initialization (random non-negative values for matrices $A$ and $S, 30$ draws per depth): unmixing processing improves detection (see figure 3 - b). Finally NMF algorithm with this time Gaussian initialization for $S$ (Gaussian models are translated on a $100 \mathrm{~nm}$ range) is tested: even with less appropriate initialization (in that case when both simulation spectra are translated $50 \mathrm{~nm}$ up that simulation models for initialization), contrast is considerably improved compared to both prior cases (see figure 3 c).

From that example, we show initialization choice is very sensitive and may be unexpected, but necessary to improve markers detection. Even if an approximate initialization already improves contrast between tumoral area and healthy tissue compared to the algorithm with random initialization (see figure 3), a more accurate initialization selection may push back the detection limits. Such selection can be obtained with a multi-start initialization step prior to the classical NMF algorithm [4] which finds the most appropriate initial candidates for matrices $A$ and $S$ : the initial estimates from a defined range 


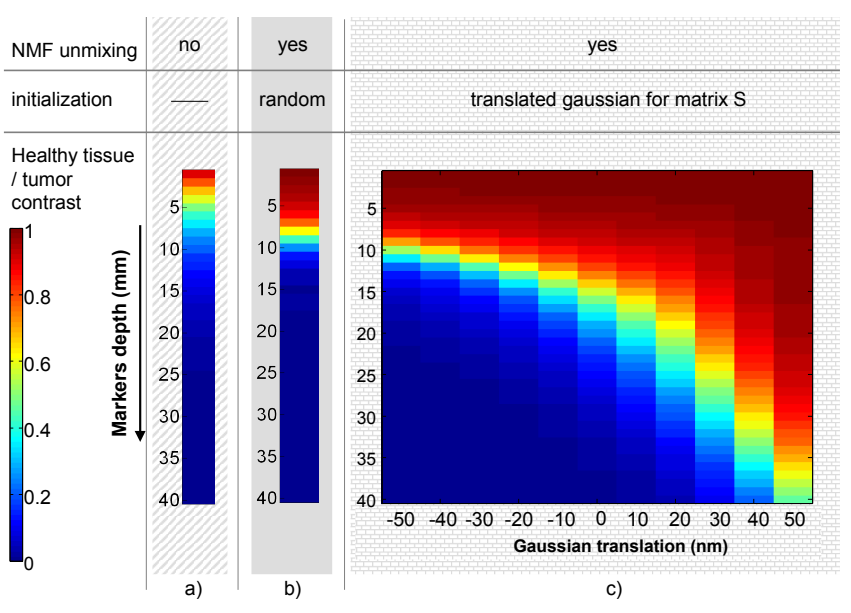

Fig. 3. Influence of initialization on resulting contrast (breast simulation example).

of initialization which give the lowest value of healthy tissue/tumor contrast or cost function $F$ after a few iterations are expected to be the most suitable for carrying on the optimization step.

\subsection{Sparsity constraints}

An other regularization we considered for our unmixing problem is based on spatial sparsity constraints.

Indeed, for local specific markers distribution (only around the tumor), we expect to get peaked weight columns in matrix $A$. A contrario, when unmixing is not complete, a residual autofluorescence baseline surrounds the specific marker peak. This property is depicted figure 3.4 .

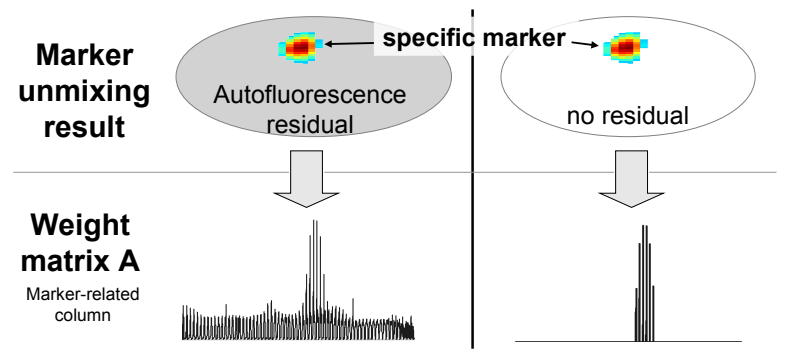

(a) Inaccurate unmixing

(b) Correct unmixing

Fig. 4. Link between the quality of unmixing process and resulting weight matrix $A$.

An intuitive reasoning to improve unmixing would be to smooth over the unwanted autofluorescence residuals on specific markers weight profiles, by thresholding the smallest values: in other words, we look for sparse $A$ columns for specific markers contributions.

We introduce a sparsity measurement [7]: let us consider a weight matrix $A$ of size $X \times P$, the sparsity of a given column $A_{p}$ $(p \in(1, P))$ of $A$ is:

$$
\text { sparsity }\left(A_{p}\right)=\frac{\sqrt{X}-\left(\sum_{x=1}^{X}\left|a_{x p}\right| / \sqrt{\sum_{x=1}^{X} a_{x p}^{2}}\right)}{\sqrt{X}-1}
$$

Sparsity value ranges from 0 for non-sparse results to 1 for extremely sparse results. Existing algorithms [7] propose from a chosen sparsity value to find a solution with exactly the same sparsity value. Since sparsity value of markers column can not be exactly known, we prefered to deal with thresholding. We also choose a sparsity value $\varphi$, and our methopd looks for most appropriate threshold value in order to find a solution whose saprsity value remains close to $\varphi$. Nevertheless, the minimization of $F$ alternatively will prevent the NMF solution to remain exactly equal to the sparsity set point. The following NMF implementation has been developped in order to take sparsity constraints into account:

Algorithm: NMF with sparsity constraints

1 to 3: idem classical NMF implementation

4. Each column $A_{p}$ of $A$ referring to weights of specific markers, for a wanted sparsity value $\varphi$ for coefficients of column $A_{p}$, is changed into $\tilde{A}_{p}$ :

$$
\forall x \in(1, X) \text {, }
$$

$$
\tilde{a}_{x p}=\left\{\begin{array}{cl}
a_{x p} & \text { if } \quad a_{x p} \geq \frac{\max \left(a_{x p}\right)}{\beta_{p \min }} \\
0 & \text { otherwise }
\end{array}\right.
$$

with $\beta_{\text {min }}$ the threshold value such as:

$$
\beta_{\text {pmin }}=\underset{\beta_{p}}{\operatorname{argmin}}\left|\operatorname{sparsity}\left(\tilde{A}_{p}\left(\beta_{p}\right)\right)-\varphi\right|
$$

5. restart steps 2 to 4 until stopping criterion is obtained (for example when $F=\epsilon$ for a chosen $\epsilon$ ).

6. At last iteration, run again steps 2 and 3 .

Finally, when running this new algorithm on simulated data presented earlier, we considerably improve tumor detection, specially for deep embedded tumors. Figure 5 gives contrast values between tumor and healthy tissue depending on tumor depth obtained when running different NMF method (with or without regularization) on breast example. On that particular example, coupling sparsity constraints to multi-start initializations, allowed to get a contrast value equal to 0.6 for example for markers $3 \mathrm{~cm}$ deep in tissues. Without NMF processing, detection with a contrast equal to 0.6 was obtained for markers only $2 \mathrm{~mm}$ deep in tissues. Finnaly NMF processing without any regularization improved detection results compared to the no processing case, with a contrast of 0.6 at $12 \mathrm{~mm}$, far from the $3 \mathrm{~cm}$ offered by sparsity constraints and multi-start initialization. By selecting accurate regularization for the unmixing problem, one may push the tumor detections limits back of a few centimeters, which is not negligible for clinical applications.

\section{IN VIVO RESULTS}

In order to simulate marked tumors on a mouse, two glass capillary tubes filled with respectively $5 \mu \mathrm{l}$ of Indocyanine Green loaded into nanoparticles (ICG-LNP) [8] at $0.35 \mu \mathrm{mol} / 1$ and with $5 \mu \mathrm{l}$ of Alexa 750 at $0.1 \mu \mathrm{mol} / 1$ are inserted subcutaneously on the animal (see figure 6). Low markers dosage are chosen, in order to emphasize autofluorescence problem and to reproduce deep tumor detection in human organs.

The resulting intensity scanning is presented figure 6 . On that precise example, we run the NMF algorithm with the multi-start initialization step and the sparse NMF iterations (initial sparsity value is 


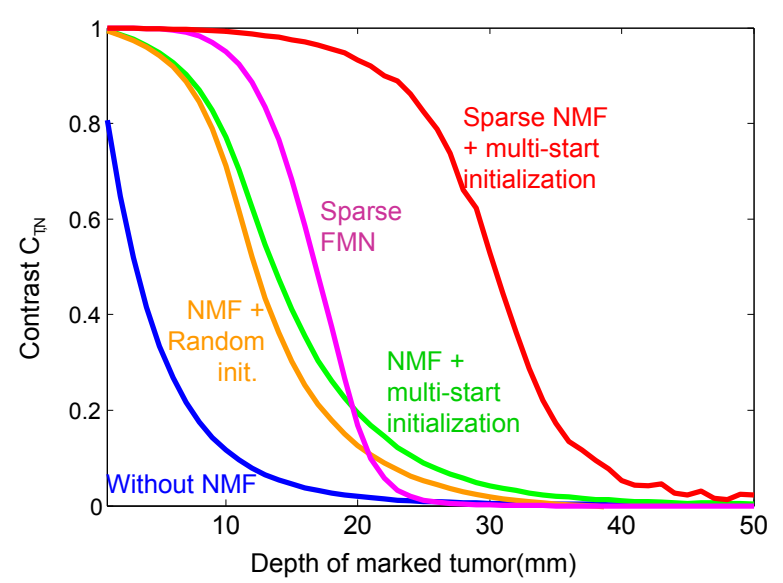

Fig. 5. Contrast $C_{T, N}$ between tumor and healthy tissue, depending on tumor depth, and NMF method. Accurate regularization on NMF may improve detection and tumor localization of a few centimeters in tissues.

chosen equal to 0.9 for both specific markers weight vectors). Mixed data size (matrix V) is $255 \times 1024$, and the NMF run-time is less than a minute.

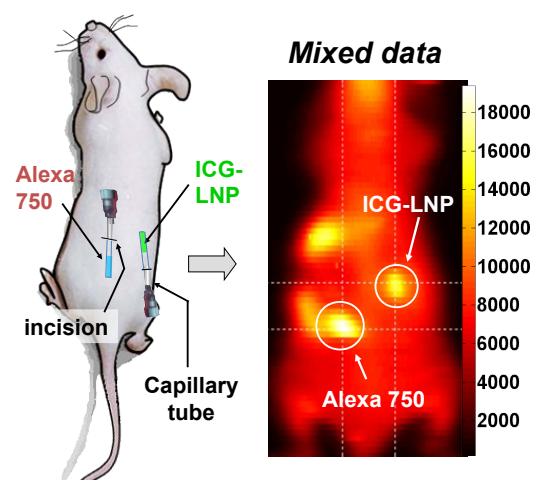

Fig. 6. Experiment on mouse; left: two capillary tubes filled with fluorescent markers simulate marked tumors, right: mixed fluorescence acquisition obtained

Figure 7 presents the unmixing results: three fluorescence spectra have been separated (figure 7-left) and the unmixed fluorescence distrbution are presented figure 7. NMF under sparsity constraints successfully unmixed the three components, and considerably improved both simulated tumors detection.

\section{CONCLUSION}

Beyond the specific fluorescence signal of specific markers used in optical imaging, the autofluorescence of biological tissues needs to be removed to get accurate detection results. To unmix fluorescence spectra, Non-negative Matrix Factorization method was chosen, and regularized algorithm were proposed to restrain the non-uniqueness ambiguity of separation. Simulated results and in vivo mice results underlined accurate regularization interest on tumor detection improvement. Autofluorescence removal as a preprocessing step for
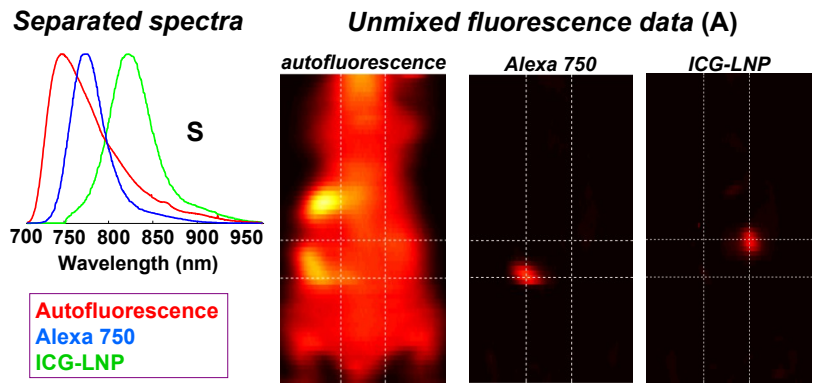

Fig. 7. Unmixing results after NMF with sparsity constraints and multi-start initialization.

fluorescence optical imaging data is more than necessary to get correct 3D tomographic reconstructions, and to accurately localize tumors.

\section{REFERENCES}

[1] V. Ntziachristos, E.A. Schellenberger, J. Ripoll, D. Yessayan, E. Graves, A. Bogdanov, L. Josephson, and R. Weissleder, "Visualization of antitumor treatment by means of fluorescence molecular tomography with an annexin v-cy5. 5 conjugate," Proceedings of the National Academy of Sciences, vol. 101, no. 33, pp. 12294, 2004.

[2] D. D. Lee and H. S. Seung, "Algorithms for non-negative matrix factorization," Advances in neural information processing systems, vol. 13, pp. 556-562, 2001.

[3] S. Moussaoui, D. Brie, and J. Idier, "Non-negative source separation: range of admissible solutions and conditions for the uniqueness of the solution," in Proceeding to IEEE International Conference on Acoustics, Speech, and Signal Processing, March 2005, vol. 5, pp. 289-292.

[4] A. Cichocki, S. Amari, A.-H. Phan, and R. Zdunek, Nonnegative Matrix and Tensor Factorizations: Applications to Exploratory Multi-way Data Analysis and Blind Source Separation, WileyBlackwell, Chichester, UK, 2009.

[5] A-S. Montcuquet, L. Hervé, F. Navarro, J-M. Dinten, and J. I. Mars, "Nonnegative matrix factorization: a blind spectra separation method for in vivo fluorescent optical imaging," J. Biomed. Opt., vol. 15, no. 5, pp. 056009-1 - 056009-14, 2010.

[6] Z. H. Jin, V. Josserand, S. Foillard, D. Boturyn, P. Dumy, M. C. Favrot, and J. L. Coll, "In vivo optical imaging of integrin alphav-beta3 in mice using multivalent or monovalent crgd targeting vectors," Molecular Cancer, vol. 41, no. 6, pp. 1-9, 2007.

[7] P. O. Hoyer, "Non-negative matrix factorization with sparseness constraints," The Journal of Machine Learning Research, vol. 5, pp. 1457-1469, 2004.

[8] F. P. Navarro, M. Berger, M. Goutayer, S. Guillermet, V. Josserand, P. Rizo, F. Vinet, and I. Texier, "A novel indocyanine green nanoparticle probe for non invasive fluorescence imaging in vivo," Progress in biomedical optics and imaging, vol. 10, no. 30, pp. 1-10, 2009. 